Apuntes Universitarios, 2022: 12(1), enero-marzo ISSN: 2304-0335 DOI: https://doi.org/10.17162au.v12i1.724

\title{
Hacia un perfil de la figura del asesor de investigación en el posgrado
}

\author{
Towards a profile of the figure of the postgraduate research advisor
}

\author{
Claudia Alejandra Bejarano Confalonieri ${ }^{1}$, Edwin Roger Esteban Rivera ${ }^{2 a}$ \\ Universidad Pontificia de Salamanca UPSA, Salamanca, España ${ }^{1}$ \\ Universidad Nacional Hermilio Valdizán, Huánuco, Perú ${ }^{2}$ \\ (iD) ORCID: https://orcid.org/0000-0003-4565-9987 ${ }^{1}$ \\ (iD) ORCID: https://orcid.org/0000-0003-4669-1268²
}

Recibido: 14 de mayo de 2021

Aceptado: 12 de agosto de 2021

\section{Resumen}

El propósito del artículo es doble: realizar un acercamiento a las palabras con que se designa a quienes guían al posgraduando en el proceso de investigación conducente a obtener el grado académico; y proponer aspectos a considerar en el perfil del asesor. Por ello, se recurrió al análisis de contenido cualitativo de documentos normativos universitarios, libros y artículos de revistas indizadas en base de datos SCOPUS, Web Of Science, SciELO, entre otros. Se establecieron tres categorías: 1, análisis semántico del asesor de investigación; 2, asesoría de tesis en los programas de posgrado; 3, elaboración del perfil de asesor de tesis. Se concluye que, en los estudios de posgrado, es imprescindible la asesoría en la formación de futuros investigadores; por ende, se debe emprender la formación de asesores a fin de promover el saber, la integración a redes de colaboración y el surgimiento de nuevos referentes en la generación, innovación y transferencia de conocimientos. Asimismo, se espera que los asesores muestren empatía, que posean actitudes, habilidades y disposición para llevar a cabo su labor con efectividad, en el marco del respeto y sentido ético.

Palabras clave: Posgrado; universidad; asesor de tesis; investigación; tutor, educación.

\begin{abstract}
This article has a twofold purpose: first, to make an approach to the words used to designate those who guide the graduate in the research process leading to obtaining the academic degree; second, to propose aspects to be considered in the profile of the advisor. Therefore, the qualitative content analysis of university normative documents, books and journal articles indexed in the SCOPUS database, Web Of Science, SciELO, among others, was used. Three categories were established:
\end{abstract}

${ }^{\mathrm{a} C}$ Correspondencia al autor:

edroer@gmail.com 
1 , semantic analysis of the one that guides the research work; 2 , thesis advice in postgraduate programs; 3, making of a thesis advisor profile. It is concluded that in postgraduate studies counseling is essential for the training of future researchers; therefore, the training of advisers should be undertaken to promote knowledge, integration into collaboration networks and the emergence of new references in generation, innovation and knowledge transfer. Likewise, advisers are expected to show empathy, who possess attitudes, skills and disposition to carry out the advice effectively within the framework of respect and ethical sense.

Keywords: Postgraduate; university; thesis advisor; research; tutor, education.

\section{Introducción}

La universidad, como institución educativa del más alto nivel en el mundo, posee tres funciones básicas: hacer docencia, promover la investigación y cumplir su vinculación con una sociedad que, a pesar del tiempo transcurrido, se mantiene en esencia (Ortega, 1992; Soledispa et al., 2021). De estas tres funciones, es la promoción de la investigación la que marca la diferencia entre la universidad y otras instituciones del nivel superior. En este caso, Giner (1990) precisa que, desde un principio y en diferentes niveles, las universidades sirven como catalizadores de la investigación científica como vía para el descubrimiento de la verdad, la que se incorpora al sistema de su cultura general y, desde allí se produce la difusión de la cultura, de sus percepciones, sentimientos e ideales, mediante la enseñanza.

En tiempos actuales, las universidades ofrecen dos niveles de formación: pre y posgrado. El pregrado se orienta a la formación básica y el posgrado se orienta a la especialización y profundización en la investigación científica. La formación de investigadores en estudios de posgrado adquiere relevancia en la sociedad del conocimiento, debido al crecimiento cuantitativo de los mismos en América del Sur como en otros continentes. Por ello, Piñero et al. (2021) sostienen que, desde hace varios años, se percibe una creciente demanda por los estudios de posgrado, incrementándose el número de estudiantes, aunque se carece del aporte científico de las investigaciones, y con ello se produce un empobrecimiento de la calidad educativa.

Con el propósito de desarrollar las competencias investigativas y disciplinares de sus estudiantes, las escuelas de posgrados contemplan la realización de investigaciones durante el proceso formativo. Al culminar sus estudios, los maestrandos y doctorandos deben presentar por escrito una tesis o artículo científico publicado en revista indizada y, exponer ante un jurado evaluador (UNHEVAL, 2017; UNSA, 2019; UNTUMBES, 2017). Para que esto sea efectivo, es 
primordial contar con un docente que asuma la función de asesor durante el proceso de investigación, redacción del informe y publicación del trabajo final. Tradicionalmente, es un profesor que, individual o dualmente (con un coasesor), se responsabiliza de guiar al estudiante en la elaboración del estudio. No obstante, la función de los asesores no es sencilla, existen muchos obstáculos_como las políticas institucionales y el aumento de la carga de trabajo (Varieur et al., 2021). Los obstáculos se incrementan si no se cuenta con instrumentos que orienten su desempeño, de ahí que, en algunos casos, el quehacer del asesor se regula por sus propias experiencias, saberes y creencias, careciendo de procesos de evaluación y reflexión de su praxis (De la Cruz et al., 2010).

Frente a esta realidad, la gestión de trabajos académicos de posgrado, como práctica educativa, formal y sistemática, es factor primordial en la formación de nuevos investigadores y del éxito del estudiante en el programa de posgrado. Cabe señalar que la deserción y el bajo índice de estudiantes graduados se constituyen en una problemática para cualquier universidad en su dimensión institucional, entre éstas, la de su calidad educativa (Fernández \& Wainerman, 2014).

En este panorama, son muchos los factores para que los estudiantes no concluyen la investigación, sean motivos internos o externos que impidan su realización, siendo lo más habitual la falta de una asesoría adecuada que les permita finalizar su trabajo final de posgrado en el tiempo establecido (Santa Cruz \& Duran, 2018). Esta problemática reporta gran interés por su tratamiento e investigación, la cual lleva a explicitar algunas preguntas como ejes ordenadores: ¿Qué se entiende por asesor? y ¿cuáles son los rasgos que deben definir a la figura del asesor de investigación en los programas de posgrado? Por ello, el propósito de esta investigación es realizar un acercamiento a las palabras con que se designa a quienes guían al posgraduando en el desarrollo de la tesis, y proponer aspectos a considerar en el perfil del asesor de tesis en los programas de posgrado.

\section{Metodología}

La investigación se apoya en el paradigma interpretativo, el mismo que se constituye en base para la perspectiva metodológica cualitativa (Piñero et al., 2019) que permite analizar e interpretar diversos documentos en formato físico y virtual que abordan la asesoría en los programas de posgrado. En este contexto, se analizaron documentos normativos de universidades, libros y artículos de revistas indizadas en base de datos SCOPUS, Web Of Science, SciELO, entre otros, que nos brindan información confiable y actualizada. Los descriptores de búsqueda fueron: 
“asesor de tesis,” “director,” “tutor,” “mentor,” “consejero” y “thesis advisor." Se empleó el análisis de contenido cualitativo, que consta de los siguientes procedimientos: Selección del objeto de análisis, preanálisis, definición de las unidades de análisis, codificación, categorización y hallazgos (Cáceres, 2003).

\section{Análisis conceptual de términos relacionados al asesor de investigación en posgrado}

El término para designar a quien guía a los estudiantes en los trabajos de investigación, incluyendo la tesis, puede variar de una universidad a otra, por ejemplo: director de investigación, consejero, asesor, tutor, mentor, etc. En este caso, se estima pertinente describir brevemente y a modo ilustrativo, en una necesaria clarificación conceptual de los términos con los que se suele nombrar a quien se desempeña en esta actividad académica en el nivel de posgrado.

\section{Director de tesis}

Es la persona con la máxima responsabilidad en la orientación de las actividades investigativas del estudiante. Puede desempeñarse como director de tesis cualquier profesional de reconocida trayectoria en investigación y con título de posgrado. Su figura es obligatoria en el estudio de posgrado, establecido por los reglamentos o normativas vigentes universitarias. Por ejemplo, en la Universidad Veracruzana de México se concibe como director de tesis al académico que orienta a los estudiantes en la realización de la investigación, redacción del informe y sustentación oral, conducentes al grado académico (Universidad Veracruzana, 2010).

\section{Tutor}

Este término posee varios significados en el campo de las ciencias de la educación; sin embargo, se le vincula más a la orientación educativa (Gonzáles \& Vélez, 2014; Aguilera, 2019). Frecuentemente, se emplean como sinónimos de “tutoría” a “tutela,” “guía," “asesoramiento,” “orientación,” “ayuda” y “asistencia” (Lázaro, 1997; Gairín et al., 2013).

El tutor es un docente del plana titular de una universidad, generalmente psicólogo, que guía al estudiante durante su estancia en la universidad a través de sesiones individualizadas, para prevenir o afrontar con éxitos problemas mentales (Vales et al., 2009). La tutoría es permanente y busca la formación integral, mayor autonomía y mejor preparación del estudiante a lo largo de la vida (Álvarez \& González, 2015). Su función no es guiar a profundidad en la tarea investigativa al 
posgraduando, sino articular su labor docente con la investigación; es decir, hacer investigación en el mismo desarrollo curricular, concibiéndose de esta manera como dualidad inseparable entre enseñanza e investigación (Aguilera, 2019).

\section{Mentor}

Es la persona con mayor experiencia en una actividad o ámbito y su correspondiente alumno—con menor o nula experiencia-recibe el nombre de mentorizado. La mentoría está orientada a promover el desarrollo de las competencias del estudiante, a fin que pueda desempeñar exitosamente las actividades como profesional (Vélaz, 2009). En algunas universidades la mentoría es ejercida por estudiantes avanzados e incluso por egresados, quienes participan como voluntarios, previa realización de una formación específica.

\section{Consejero}

El consejero es aquel docente que tiene la función de orientar y asesorar a los estudiantes en las diversas facetas inherentes a la vida académica. Las consejerías son esencialmente individualizadas o privadas, a fin de optimizar la orientación al estudiante, de modo que este pueda concluir con éxito el programa (Garzón, 2018).

\section{Asesor}

Es el encargado de orientar al estudiante en el proceso investigativo: tesis o artículo científico, velando por la calidad académica y el cumplimiento de los aspectos éticos legales; es decir, el asesor brindando el soporte adecuado en la fundamentación ontológica, epistémica, metodológica y axiológica de la investigación (Piñero et al., 2019). La labor del asesor es formativa, sistemática y cualitativa, desde la elección del problema o tema hasta la sustentación y publicación de los resultados de la investigación.

\section{Análisis general del rol de asesor de trabajos de investigación de posgrado}

A la luz del análisis conceptual de la figura del docente que asume el rol de conducir la producción de trabajos en posgrado, se fue abriendo la temática a un panorama mucho más amplio y profundo. Cada uno de los rasgos o aspectos que lo conforman y los numerosos aportes que lo 
caracterizan, otorgan una accesibilidad, en su amplia connotación de apertura al conocimiento, desde cualquiera de sus múltiples facetas para pensarlas y estudiarlas.

En los términos que se presentaron en párrafos anteriores, se observa que tales significados se alternan y conjugan con sus formas, las cuales sugieren un movimiento semántico con sus ondulaciones de significado. Dependiendo del documento que se consulte, se hace referencia a mentores, tutores, asesores, directores de investigación u otra denominación análoga. Sin embargo, ya sea que se hable de cualquiera de estos términos, se trata de una figura que es considerada como modelo, guía, líder o facilitador que busca ayudar al estudiante de posgrado en el proceso de investigación conducente al grado académico. Por ende, en cuanto a la figura del asesor, que como objeto de estudio sigue en construcción y evolución permanente, se afirmar que:

a) Constituye para la educación de nivel superior actual, un motivo de ocupación por todo lo que implica.

b) Se requiere tener claro una conceptualización sobre esta figura docente, que ejerce su función ante una diversidad de características de los estudiantes. Las asesorías deben responder a la heterogeneidad de necesidades académicas investigativas de los estudiantes, a sus formas de pensamiento, modos de aprender, sus habilidades sociales, comunicativas, intereses, motivos y sus talentos, que completan su trayectoria académica.

\section{La asesoría de tesis en los programas de posgrado}

A pesar que existen diversos términos para designar al que guía el proceso de investigación, de aquí en adelante emplearemos el término asesor. La masificación de los estudios de posgrado trastoca la noción tradicional de una buena asesoría. Un "buen” asesor no puede aconsejar a un número elevado de estudiantes en el mismo periodo; por ello, estos recurren a maestros o doctores que no cuentan con las competencias requeridas, conscientes que en América Latina no se exige preparación como sí ocurre, por ejemplo, en Austria (Manathunga, 2005). La realidad muestra que es difícil encontrar escuelas de posgrado que formen sistemáticamente asesores de investigadores.

Repasando la información disponible acerca de cómo es la asesoría en distintos lugares del mundo, se pudo encontrar que la asesoría es inherente al docente universitario, a pesar que esta actividad se desarrolla con ciertos matices entre universidad y otra. Está el caso de Alemania, donde asesores y asesorados asumen el compromiso de generar conocimientos científicos. En el Reino 
Unido, específicamente en la universidad de Oxford, el asesor trasciende por ser competente en el desarrollo del pensamiento crítico y reflexivo de sus estudiantes (Rodríguez, 2012; Lobato \& Guerra, 2014).

Bortolus (2014) considera que uno de los aspectos más importantes cuando uno asume la realización de una tesis de posgrado, es la elección de los asesores. A pesar de lo importante de esta labor académica, las personas no logran planificarla con el debido cuidado. Las consecuencias de una mala elección pueden verse en los numerosos foros que en diversos países del mundo se dedican a dar apoyo a estudiantes con serios problemas de asesoría; situaciones que van desde el ámbito académico, hasta el profesional e incluso el legal.

Elegir un asesor requiere la reflexión sobre el perfil que este tenga. Ubertonef (2011), en este sentido, plantea que la elección de un asesor debe considerarse en base a un conjunto de criterios, entre ellos revisar los reglamentos, confirmar si el asesor cumple con lo requerido en el reglamento, reflexionar por la relación armoniosa que podría existir entre estudiante y asesor, evaluar si el asesor tiene tiempo para atendernos, si tiene experiencia en guiar procesos de investigación, etc.

Desde otro aspecto, la labor del asesor va más allá de la culminación de la tesis o artículo científico del asesorado, su labor es también contribuir a la formación de nuevos investigadores con condiciones para formar parte de una comunidad investigativa (Wenger, 2001). En esta línea, según De la Cruz et al. (2010), se requiere contar con asesores que posean competencias formativas-socializadoras y competencias interpersonales. Las primeras exigen que los asesores deben estar en condiciones de capacitar a los estudiantes para que realicen investigaciones originales de manera autónoma, contribuyendo a la solución de problemas vinculadas a su profesión. Las segundas requieren asesores con comportamiento ético, capaces de generar un clima de confianza y empatía en la interrelación asesor-asesorado. Por ello, al revisar las funciones del asesor de tesis en el posgrado (tanto en Perú cómo en Argentina), no se encuentra un manual específico del asesor. Los reglamentos de manera genérica mencionan las funciones del asesor; sin embargo, no resulta claro los roles específicos de los asesores y se obvia el sistema de evaluación de la práctica de las asesorías (Hernández \& Martínez, 2016).

De igual manera, Ubertone (2001) narra que un profesor universitario estudiante del doctorado, eligió como asesor de tesis a un sobresaliente docente de su Facultad. Resulta que él quería investigar un tema y el asesor otro. Al final, no se pusieron de acuerdo, el estudiante se 
desanimó y nunca realizó la tesis doctoral. Frecuentemente, el profesor recordaba con pena este suceso que marcó su vida; por lo que esta anécdota brida lecciones para que los estudiantes no sufran frustraciones por la misma causa. Es muy importante que exista una instancia con la correspondiente reglamentación para evaluar el trabajo que realizan los asesores, con el objetivo de garantizar un sistema de asesoría de calidad.

En este caso, existen ciertos factores que influyen de manera negativa en el desarrollo de una adecuada asesoría, entre ellos destacan: a) La carencia de estímulos al asesor por el tiempo que dedica a la asesoría; b) la falta de programas de fortalecimiento de capacidades a los asesores que vaya más allá de cómo realizar una tesis; c) carencia de espacios para discutir logros y dificultades halladas en las asesorías; d) actitud del asesor en que el estudiante de posgrado no requiere de orientación para hacer una buena investigación, que "él puede solo”; e) Los asesores a menudo no disponen del tiempo para realizar un monitoreo adecuado, tampoco para prepararse a fin de realizar un adecuado la monitoreo, tampoco tienen tiempo para recibir el apoyo adecuado.

\section{Hacia un perfil del asesor de tesis en posgrado}

Las universidades nacen en el desarrollo histórico de las sociedades, a su vez, las universidades desempeñan un importante rol en el desarrollo de esas sociedades (Esteban, et al., 2021); por ende, las universidades están llamadas a garantizar la formación de maestros y doctores capaces de enfrentar con éxito los retos presentes y venideros. Además, la sociedad exige a la universidad que genere conocimiento, a fin de resolver problemas del contexto, esta tarea solo es posible mediante la investigación. El conocimiento es muy preciado en la actualidad a tal punto que se le denomina capital intelectual, capital cultural (Piñero et al., 2021) y, la propiedad del conocimiento se ha expandido en el mundo.

Las exigencias que hace la sociedad a la universidad y sobre a los posgrados, se incrementa por el COVID-19 y factores asociados, a decir de Piñero et al. (2021), y esto ahora exige que los programas de posgrado cumplan su rol como estudios del más alto nivel, con el trabajo de resolver esos problemas actuales a través de la investigación y del trabajo colaborativo. Esto depende en gran medida del perfil del asesor y en focalizar el dominio teórico, metodológico y pedagógico del asesor; y en menor medida, a la relación armónica que debe existir entre asesor y asesorado.

\section{Dimensión profesional}

En este plano se requiere que los asesores puedan ser competentes en las siguientes áreas: 
Conocimiento de los lineamientos de la institución. El asesor debe conocer y poner en práctica los lineamientos de investigación de la institución educativa al que pertenece el asesorado. Ha de conocer el reglamento de grados, las líneas de investigación, los esquemas de investigación y las normas de redacción asumidas del posgrado.

Preparación académica. El asesor debe ser un profesional que demuestre en el plano conceptual y procedimental dominio de la metodología de investigación, que muestre experiencia para monitorear investigaciones y denote ser especialista en el área que se realiza la investigación (Rosas et al., 2006). Por ello, es de suma importancia que el asesor haya realizado investigaciones y que muestre los resultados en libros arbitrados y/o en revistas indizadas; con ello se confirma lo que aducen De la Cruz et al. (2010) corresponde a los asesores contribuir a que los estudiantes profundicen el dominio teórico de su campo profesional a la vez que desarrollen investigaciones.

Saber pedagógico. Las instituciones educativas de todos los niveles tienen un rol de primer orden en la construcción de la sociedad. Sin embargo, comúnmente se cree que lo más importante en términos educativos es el conocimiento del profesor sobre los contenidos que imparte, dejando de lado su experiencia docente; las vivencias en su trayectoria laboral; la concepción del mundo, de la vida y de educación. En suma, el saber pedagógico del docente hace posible que el mensaje que desea transmitir o la experiencia que desea promover, se logre.

Por todo lo anteriormente visto, se puede afirmar que no basta con que el docente sea un experto investigador o erudito en la materia que se está investigando; la realidad exige que se requiera su habilidad de hacer fácil de entender lo que habitualmente se considera complicado o imposible.

\section{Dimensión de relacionalidad}

En este plano se requiere que los asesores puedan evidenciar las siguientes caracteristicas:

Predisposición para la asesoría y monitoreo permanente. Realizar una investigación no es tarea sencilla, pues en el proceso de acompañamiento al estudiante, el asesor se constituye en coautor de aquella. Se requiere que asesor y asesorado tengan varias sesiones para elaborar el proyecto de estudio y todo lo que ello implica. Se precisa que el asesor destine mucho tiempo para el asesoramiento y monitoreo permanente (Difabio, 2011); y es imprescindible la predisposición del asesor, toda vez que, según Revilla (2017), la asesoría debe ser permanente para desarrollar y culminar la tesis en el tiempo establecido y con el rigor científico exigido. 
Motivador. Los asesores tienen la función de orientar al estudiante en el proceso de investigación, por lo que es inevitable que el asesorado adquiera confianza en sí mismo, y que se motive por realizar la investigación. Según Suárez (2016), la motivación del tutor repercute de manera positiva en el estudiante, no solo en los aprendizajes curriculares, sino también en el moldeamiento de su proyecto de vida. De esta forma, los asesores se adjudican un tipo de coach porque orientan, promueven la reflexión y el análisis, monitorean el trabajo de los estudiantes y los involucran, teniendo en cuanta sus subjetividades (De la Cruz \& Abreu, 2011; Mamani, 2019).

Respeto a ideas del asesorado. Al momento de empezar una investigación, es normal que surjan algunas interrogantes: ¿Qué tema investigar?, ¿Cómo investigar? De esta elección depende la culminación o abandono del trabajo, por lo que al investigador le debe agradar el objeto de estudio. En este caso, el trabajo del asesor es brindar un conjunto de opciones con relación al objeto de estudio determinado por el estudiante, pero jamás debe orientar el estudio a sus propios intereses (Difabio, 2011). El asesor debe ilustrar sobre las características y viabilidad de los diferentes métodos con los que se puede emprender el objeto de estudio, y dejar a la elección del estudiante el método de investigación, así no sea del agrado absoluto del asesor (Revilla, 2017).

Responsabilidad. Esto se resuelve en el apoyo constante al estudiante, estructurando en conjunto las diferentes etapas de la investigación, realizar sugerencias constructivas, revisar y devolver los avances de la investigación en el tiempo establecido (Rosas et al., 2006). En este sentido, el asesoramiento significa apertura, amistad, dedicación, desprendimiento y trabajo en equipo (Santa Cruz \& Duran, 2018)

Cabe señalar que el profesor no es el único que asume responsabilidades en la asesoría, el estudiante es responsable de su propia formación, la institución en su conjunto también es responsable. Depende del esfuerzo y del despliegue de estos tres elementos la consecución de los propósitos de la asesoría.

Empático. Con frecuencia los asesores y jurados de tesis juzgan, desde su óptica y experiencias, a los trabajos de los maestrandos y doctorandos que-como es natural—tienen menor trayectoria académica, lo que es entendible en el caso de los jurados, mas no en el caso de los asesores; estos deben ponerse en el lugar del estudiante al momento de juzgar el trabajo o en el proceso de conducción, y debe tener presente los saberes con los que cuenta el estudiante. Según Jiménez (2019) deben existir buenas relaciones entre asesor y asesorado, la que se traduce en confianza mutua. El estado emocional que se genera en el asesorado también condiciona en gran 
medida los resultados a obtener (Goleman, 1998, como se citó en Esteban et al., 2020). Por ello, un asesor empático está siempre abierto a comprender las expresiones de sentimientos de sus asesorados.

Comportamiento ético. En este caso, el asesor debe promover en los estudiantes el trabajo autónomo, respetando autorías, la persona del mismo asesorado y no aprovechar de su estatus como asesor para beneficiarse de los estudiantes (De la Cruz et al., 2010). Por ello, y con la finalidad de ofrecer el servicio de asesoría de manera adecuada que responda a las exigencias de la institución educativa y de los posgraduandos, es inevitable implementar la evaluación a los asesores. Entre los aspectos a considerar están: a) entrevistas periódicas a los asesorados sobre el grado de satisfacción; b) entrevistas periódicas a los asesores como parte de la evaluación al sistema de asesorías; c) informe de los asesores sobre el desarrollo de las asesorías, contemplando entre otros puntos, logros, dificultades, puntos de quiebre, estrategias adoptadas; d) Realización de grupos focales sobre aspectos específicos que permitan evaluar el trabajo de los asesores; e) espacios reflexivos de la propia práctica de asesoría, con referencia a las debilidades y las fortalezas auto percibidas en las tareas de asesoría.

\section{Conclusiones}

La masificación de los programas de posgrado en América Latina y el mundo no tiene relación directa con el incremento de investigaciones de impacto (Piñero et al., 2021), y la producción científica de los posgraduandos tiene serias limitaciones. En este aspecto, una de las debilidades es la escasa publicación de los resultados de las investigaciones en revistas indexadas, debido entre otros factores, a la falta de orientación adecuada del asesor de tesis, trabajo que se complica por las nuevas condiciones de vida producto de la pandemia por COVID-19. En este caso, es necesario reflexionar sobre cómo se viene realizando la asesoría en el impulso de la producción científica en el nivel de posgrado. La asesoría en los estudios de posgrado es imprescindible para la formación de futuros investigadores, adecuadamente desarrollada contribuye a generar nuevos conocimientos y a consolidar nuevos líderes capaces de generar, innovar y transferir conocimientos.

Un buen asesor debe tener experiencia académica y en investigación, factor pedagógico que facilita el aprendizaje de los asesorados, además de actitudes, habilidades, disposición para conducir con efectividad la asesoría. El asesor como persona libre, plena y digna, es aquel docente 
académicamente formado que aspira a la calidad educativa. Es un profesional que desarrolla permanentemente sus competencias de manera multidimensional en su quehacer pedagógico y docente, desde la cotidianeidad y a lo largo de la trayectoria de su carrera en cada universidad, para poder contribuir a la mejora tanto del desarrollo personal como organizacional en la complejidad del mundo en el que se vive.

\section{Referencias}

Aguilera, J. L. (2019). La tutoría universitaria como práctica docente: fundamentos y métodos para el desarrollo de planes de acción tutorial en la universidad. Pro-PosiçõesPro-Posições, 30, 1-27. https://doi.org/10.1590/1980-6248-2017-0038

Álvarez, P. R., \& González, M. C. (2015). Estrategias de intervención tutorial en la universidad: una experiencia para la formación integral del alumnado de nuevo ingreso. Tendencias Pedagógicas, 16, 237-256.

https://revistas.uam.es/tendenciaspedagogicas/article/view/1952

Bortolus, A. (2014). Ciencia con voz propia: la ciencia de elegir un director de tesis. Consejo Nacional de Investigaciones Científicas y Técnicas, Divulgación científica, 1-3. https://www.conicet.gov.ar/la-ciencia-de-elegir-un-director-de-tesis/

Cáceres, P. (2003). Análisis cualitativo de contenido: una alternativa metodológica alcanzable.

Revista Psicoperspectivas, II, 53-82.

https://www.psicoperspectivas.cl/index.php/psicoperspectivas/article/viewFile/3/1003

Caron, R., Mattos, P., \& Barboza, J. J. (2020). Dificultades para la elaboración de artículos de investigación científica en estudiantes de posgrado en salud. Educación Médica Superior, 34 (3), 1-9. http://www.ems.sld.cu/index.php/ems/article/view/1624

Cubo, L. (2005). Los manuales universitarios . En L. Cubo de Severino, Los textos de la Ciencia. Principales clases de discurso académico-científico (pp. 325-335). Comunicarte.

De la Cruz, G., Díaz, F., \& Abreu, L. F. (2010). La labor tutorial en los estudios de posgrado. Rúbricas para guiar su desempeño y evaluación. Perfiles Educativos, 32 (130), 83-102. https://doi.org/10.22201/iisue.24486167e.2010.130.20624

De la Cruz, G., \& Abreu, L. F. (2011). Tutoría en posgrado: agente mediador en la formación de guardianes de la disciplina. En P. Ducoing, Tutoría y mediación I (pp. 191-208). IISUEUNAM. 
Difabio, H. (2011). Las funciones del tutor de la tesis doctoral en educación. Revista Mexicana de Investigación Educativa, 16 (50), 935-959.

https://www.comie.org.mx/revista/v2018/rmie/index.php/nrmie/article/view/400/400

English, E., Newman, J. S. B., Cox, A., \& Bronwyn, T. W. (2021) Embodiment, place, and stance: a collaborative exploration of graduate research and mentoring. International Studies in Sociology of Education. https://doi.org/10.1080/09620214.2021.1882870

Esteban, E. R., Callupe, S. F., Rojas, A. R., \& Cámara, A. A. (2020). Empatía y rendimiento académico en estudiantes universitarios. Revista EDUCARE - UPEL-IPB, 24, 26-46. https://doi.org/10.46498

Esteban, E. R., Portocarrero, E., Rojas, A. R., Piñero, M. L., \& Callupe, S. F. (2021). La transdisciplinariedad desde el modelo educativo: una experiencia universitaria. Revista Inclusiones, 8 (1), 241-261. http://revistainclusiones.org/index.php/inclu/article/view/197

Fernández, L., \& Wainerman, C. (2014). La dirección de tesis de doctorado: ¿una práctica pedagógica? Revista Perfiles Educativos, XXXVII (148), 156-171. https://doi.org/10.22201/iisue.24486167e.2015.148

Gairín, J., Muñoz, J. L., Galán , A., Sanahuja , J., \& Fernández , M. (2013). Planes de acción tutorial para estudiantes con discapacidad: una propuesta para mejorar la calidad formativa en las universidades españolas. Revista Iberoamericana de Educación, 63, 115-126. https://doi.org/10.35362/rie630504

Garzón, C. (2018). La gestión educativa en la educación superior de cara a la consejería estudiantil. Boletín Redipe, 7 (8), 98-112. https://revista.redipe.org/index.php/1/article/view/546

Giner, F. (1990). Escritos sobre la Universidad española. Madrid: Espasa Calpe.

Gonzáles, A. M., \& Vélez, C. (2014). La acción tutorial en el sistema escolar. UNED.

Hernández, N. L., \& Martínez, R. (2016). El proceso de asesoría en los posgrados en educación en Chiapas . En M. Vegara Fregoso, \& R. Calderón García, Procesos de formación y asesoría en programas de posgrado en educación en Latinoamérica (pp. 347-362). Universidad de Guadalajara.

Jiménez, A. (2019). La redacción científica en la universidad. Visiones de los estudiantes de posgrado de La Habana. Atenas, $14 \quad$ (4), $33 \quad$ - 46. http://atenas.umcc.cu/index.php/atenas/article/view/507/780 
Lázaro, A. (1997). La acción tutorial de la función docente universitaria. Revista complutense de educación, 8 (1), 233-252. https://dialnet.unirioja.es/servlet/articulo?codigo=150210

Lobato, C., \& Guerra, N. (2014). Las tutorías universitarias en el contexto europeo. Revista Orientación y Sociedad, 14. https://revistas.unlp.edu.ar/OrientacionYSociedad/article/view/8349

Mamani, O. J. (2019). El asesor de tesis como Coach: una alternativa para impulsar la producción científica estudiantil. Revista Cubana de Educación Médica Superior, 33 (1), 1-13. http://www.ems.sld.cu/index.php/ems/article/view/1590

Manathunga, C. (2005). Early warning signs in postgraduate research education: a different approach to ensuring timely completions. Teaching in Higher Education, 10 (2), 219-233. https://doi.org/10.1080/1356251042000337963

Narvaja, E. (2009). Escritura y producción de conocimiento en las carreras de posgrado. Santiago: Arcos.

Ortega y Gasset, J. (1992). Misión de la universidad. Madrid: Alianza.

Piñero, M. L., Esteban, E. R., Rojas, A. R., \& Callupe, S. F. (2021). Tendencias y desafíos de los programas de posgrado latinoamericanos en contextos de COVID-19. Revista Venezolana de Gerencia, 26 (93), 123-138.

https://produccioncientificaluz.org/index.php/rvg/article/view/34972

Piñero, M. L., Rivera, M. E., \& Esteban, E. R. (2019). Proceder del investigador cualitativo: Precisiones para el proceso de investigación. Lima: Fabriray.

Revilla, D. (2017). Expectativas y tensiones en la asesoría de tesis en la Facultad de Educación de la Pontificia Universidad Católica del Perú. REDU. Revista de Docencia Universitaria, 15 (1), 277-303. https://doi.org/10.4995/redu.2017.6057

Rodríguez, S. (Ed.). (2012). Manual de tutoría universitaria. Barcelona: Octaedro.

Rosas, A. K., Flores, D., \& Valarino, E. (2006). Rol del tutor de tesis: Competencias, condiciones personales y funciones. Investigación y Postgrado, 21 (1), 153-185. https://dialnet.unirioja.es/descarga/articulo/2309849.pd

Santa Cruz, F. F., \& Duran, K. L. (2018). Significado de las prácticas tutoriales en las asesorías de tesis de los estudiantes de maestría en educación. Páginas de Educación, 199-214. https://revistas.ucu.edu.uy/index.php/paginasdeeducacion/article/view/1634/1612 
Swales, J. M. (1990). Genre analysis: English in academic and research settings. New York: Cambridge University Press.

Soledispa, X. E., Sumba, R. Y., \& Yoza, N. R. (2021). Articulación de las funciones sustantivas de la educación superior y su incidencia en las competencias de la formación del profesional. Dominio de la Ciencia, 7 (1), 1009-1028.

https://dominiodelasciencias.com/ojs/index.php/es/article/view/1753/349

Ubertonef, F. P. (2011). Elegir una tesis. Revista Academia, 9 (18), 193-206. http://www.derecho.uba.ar/publicaciones/rev_academia/revistas/18/elegir-director-detesis.pdf

UNHEVAL. (2017). Modelo educativo. Huánuco: Universidad Nacional Hermilio Valdizán.

Universidad Veracruzana. (2010). Regramento general de estudios de posgrado. Veracruz, México.

https://www.uv.mx/legislacion/files/2012/12/reglamento_general_de_estudios_de_posgra do2010.pdf

UNSA. (2019). Reglamento de la Escuela de Posgrado de la Universidad Nacional San Agustín. Arequipa. http://posgrado.unsa.edu.pe/wp-content/uploads/2020/01/REGLAMENTO-DELA-ESCUELA-DE-POSGRADO-2019.pdf

UNTUMBES. (2019). Reglamento de tesis para pregrado y posgrado de la Universidad Nacional de Tumbes. Tumbes: http://www.untumbes.edu.pe/vinvestigacion/docs/31.4\%20\%20ReglamentoTesis.pdf.

Vales, J., Ramos, D., \& Olivares, K. M. (2009). La función del tutor en ambientes presenciales y no presenciales. Revista Mexicana de Orientación Educativa, 6 (16), 16-19. http://pepsic.bvsalud.org/pdf/remo/v6n16/v6n16a04.pdf

Varieur, J. A., Boyd, L. D., \& Smallidge, D. (2021). Dental hygiene faculty perspectives on thesis advising in graduate education. Journal of Dental Education, https://doi.org/10.1002/jdd.12612. doi:https://doi.org/10.1002/jdd.12612

Vélaz, C. (2009). Competencias del profesor-mentor para el acompañamiento al profesorado principiante. Proesorado, Revista de currículum y formación del profesorado, 13 (1), 209229. https://recyt.fecyt.es/index.php/profesorado/article/view/42165

Wenger, E. (2001). Participación y no participación. En E. Wenger, Comunidades de práctica. Aprendizaje, significados e identidad (pp. 205-213). Paidós. 